\section{¿MÁS ALLÁ DE LA CIENCIA ACADÉMICA?: MODO 2, CIENCIA POSACADÉMICA Y CIENCIA POSNORMAL}

\author{
María Jiménez-Buedo \\ Universidad Nacional de Educación a Distancia (UNED) \\ mariajimenezbuedo@gmail.com
}

Irene Ramos Vielba

Instituto de Estudios Sociales Avanzados (IESA-CSIC) iramos@iesa.csic.es

\section{BEYOND ACADEMIC SCIENCE?: MODE 2, POSTACADEMIC SCIENCE \& POSTNORMAL SCIENCE}

\begin{abstract}
The article carries out a revision of three theoretical perspectives that aim at describing the changes in technological and scientific research that have taken place in the second half of the twentieth century. The three approaches analysed -"Mode2", "post-academic science" and "post-normal science"-describe the transformation of science towards having a greater permeability for societal demands and preferences. These perspectives have had a great impact both in the specialized literature and in the rhetoric of $R \& D$ policy-makers. In this work, a joint analysis of its main traits is undertaken in view of providing a critical assessment of the contribution of these three approaches.
\end{abstract}

KEY WORDS: Academic science, Mode 2, Post-academic science, Post-normal science.

\section{INTRODUCCIÓN}

Al abordar los cambios más recientes en la investigación científica y tecnológica, han sido varios los enfoques que han tomado como elemento central de estos procesos una nueva y compleja configuración de las relaciones entre academia, empresas y gobierno/sociedad. El reajuste en las relaciones entre estos ámbitos tiene su origen en la crisis energética de los años setenta del siglo XX y las presiones presupuestarias a las que los gobiernos occidentales han tenido que enfrentarse en las décadas de los ochenta y los noventa.

En ese contexto de cambio se han generado distintas corrientes de pensamiento que intentan dar cuenta de formas muy distintas del papel de la ciencia y la tecnología
RESUMEN: El artículo realiza una revisión de tres perspectivas que intentan describir los cambios producidos en la segunda mitad del siglo XX en la investigación científica y tecnológica. Los tres enfoques analizados -"Modo 2", "ciencia posacadémica" y "ciencia posnormal"- asocian estas transformaciones a una mayor permeabilidad de la ciencia hacia los intereses y preferencias de los principales actores sociales. Estos enfoques han tenido un gran impacto, no sólo en la literatura especializada, sino también en la retórica de los hacedores de las políticas de I+D en los últimos años. En este trabajo se aborda un análisis conjunto de sus aportaciones más destacables y sus principales rasgos con el fin de realizar un balance crítico.

PALABRAS CLAVE: Ciencia académica, Modo 2, ciencia posacadémica, ciencia posnormal.

en la sociedad y en el desarrollo económico. De un lado, el énfasis en los riesgos asociados a los avances científicos (e.g. el impacto de la innovación tecnológica para el medio ambiente o la posible deriva armamentística de algunos desarrollos científicos) y las nuevas desigualdades económicas asociadas al desarrollo tecnológico dan lugar al surgimiento de movimientos críticos dentro de las disciplinas tradicionales de las ciencias sociales, entre los que destaca la corriente de los Estudios de Sociales de la Ciencia (Fuller,1993). De otro lado, la crisis económica también provoca que gobiernos y empresas acudan a la ciencia y la tecnología en busca de un soluciones a la dependencia energética, que en ocasiones se entienden como una panacea frente a la recesión económica y el crecimiento del desempleo (Shin, 2002). A partir de este período de transformación de la actividad de I+D y de 
efervescencia de ideas respecto a su situación y sus funciones es desde donde hay que entender las interpretaciones sobre la transformación de la ciencia y la tecnología que se tratan en este artículo.

Diversos estudios provenientes de la sociología, la economía y la filosofía de la ciencia realizados durante los setenta y los ochenta (Leydesdorff, L. y Etzkowitz, H., 1996) van coincidiendo en una visión de la ciencia como un sistema dinámico que depende no sólo de factores internos a sus organizaciones y al quehacer de los científicos, sino también por determinantes externos de carácter social o político. Al mismo tiempo, se van abandonando progresivamente los modelos que conciben la relación entre ciencia básica, aplicada y desarrollo tecnológico de una forma lineal debido a que no son capaces de dar cuenta de la verdadera complejidad de las vinculaciones multidireccionales entre estos ámbitos. Todas estas cuestiones aparecen de trasfondo en la pretensión de describir el cambio estructural producido en la investigación científica y tecnológica y vincularlo a los cambios sociales. A esa tarea han dedicado sus esfuerzos los autores que han dado lugar a los tres enfoques sometidos a análisis en este artículo -Modo 2 , ciencia posacadémica y ciencia posnormal-. Estas tres perspectivas han adquirido una importancia notable en la reflexión teórica y muchas de sus categorías de análisis han conseguido permear el ámbito de las políticas de I+D. Los tres enfoques analizados convergen en señalar el fin de un período clásico en la manera de hacer y gestionar la ciencia, lo cual ha dado paso a un sistema en el que las demandas y los controles sociales sobre los resultados científicos son mucho más fuertes. De ahí el interés por abordar conjuntamente sus contenidos y principales rasgos desde una revisión crítica en el momento actual que permita valorar sus fortalezas y puntos débiles.

El artículo aborda cada uno de los tres enfoques, haciendo un repaso detallado de los autores y obras representativas de cada uno de ellos. Esto se acompaña de una valoración de sus contribuciones más significativas al ámbito de los estudios de la ciencia y la tecnología. Se analizan los conceptos clave, las principales asunciones teóricas y sus aportaciones metodológicas. La revisión crítica permite interpretar el alcance e interrelación de sus postulados. El artículo realiza un metaanálisis sobre los tres enfoques prestando atención sus las características distintivas. Se intenta identificar qué corrientes de pensamiento inspiran las principales ideas, si éstas están formuladas como teorías explicativas y si sus enunciados se exponen de manera operativa para que puedan ser testados. También se tiene en cuenta la existencia de un componente normativo de fondo, si se pretende constituir un programa de investigación y, finalmente, si han dado lugar a cierta acumulación empírica que permita progresar a las diversas hipótesis. Las conclusiones obtenidas al ponerlos en conexión y valorarlos transversalmente posibilitan discernir su vigencia como marcos de análisis sobre la I+D y la innovación, así como su posible aplicabilidad práctica real.

\section{La NUEVA PRODUCCIÓN DEL CONOCIMIENTO}

\subsection{Características principales de la tesis del "Modo 2"}

Las tesis del "Modo 2" se recogen en dos obras clave. La primera de ellas se publicó en 1994 bajo el título The New Production of Knowledge: The Dynamics of Science and Research in Contemporary Societies, a cargo de Gibbons, Limoges, Nowotny, Schwartzman, Scott y Trow. Los autores son cientificos de diferentes paises (Inglaterra, Canadá, Austria, Brasil y Estados Unidos)'. El libro pronto adquiere gran notoriedad pública recibiendo adhesiones en ámbitos universitarios y también políticos, lo que se traslada a una importante influencia entre los gestores de sistemas científicos. El contenido gira en torno a los rasgos que caracterizan al emergente modo de producción del conocimiento que ellos denominan "Modo 2", frente al anterior "Modo 1". Pese a la rápida expansión del uso de estos términos, las críticas que recibe esta obra ponen de relieve sus ambigüedades al caracterizar el Modo 2, algunas de las cuales son reconocidas por los propios autores que -en 2001- publican un segundo libro para dotar al anterior de un mayor soporte teórico y refutar las objeciones que había recibido. En esta ocasión los responsables son Nowotny, Scott y Gibbons. Con el título Re-Thinking Science. Knowledge and the Public in an Age of Uncertainty tratan de revisar algunos de sus planteamientos previos, así como de aclarar o ampliar aspectos que habian suscitado comentarios entre los revisores de su primer libro. Además, extienden su análisis a las implicaciones del Modo 2 de producción de conocimiento para la sociedad. Esta 
nueva publicación tampoco está exenta de juicios críticos negativos. Por el contrario, suscita "nuevas perplejidades relacionadas con el concepto de sociedad implícito, las dimensiones políticas que conlleva, la carga valorativa del modelo y, más específicamente, la idea misma de ciencia a la que se refiere" (Albornoz, 2003, 225).

El Modo 1 de producción de conocimiento se identifica con la investigación tradicional, cuyo carácter es disciplinar, homogéneo y jerárquico. Es decir, la producción de conocimiento se realiza en organizaciones jerárquicas permanentes (universidades y centros de investigación) con el objetivo de avanzar en el conocimiento de la realidad para satisfacer los propios intereses académicos y disciplinarios. Sus metas son fijadas, por tanto, por los investigadores, así como el control de la calidad, que recae en manos de la comunidad de pares; tras lo cual, los resultados obtenidos pasan a ser de dominio público. El Modo 2, sin embargo, presenta todos los rasgos opuestos: es transdisciplinar, heterogéneo y heterárquico. El Modo 2 se define también por el hecho de que en él se prima la aplicabilidad y la utilidad social. La investigación en el Modo 2 traspasa los límites disciplinares, la realizan grupos no jerárquicos creados ad hoc para atender demandas sociales específicas. Se trata de una producción de conocimiento orientada al contexto de aplicación. La validación de los resultados, que son protegidos por patentes, descansa, principalmente, en la aceptación social. El cuadro 1 contrasta de manera resumida los principales rasgos diferenciadores de los dos modos de producción de conocimiento, siguiendo la aportación de Gibbons et al.

A continuación se detallan las características más determinantes del Modo 2 de producción de conocimiento:

- Contextos dinámicos de aplicación. El conocimiento del Modo 2 se produce en contextos dinámicos de aplicación. Esto significa que, primando la praxis por encima de cualquier otra consideración, se mantiene una actitud de permeabilidad a demandas y necesidades externas. De esa forma, se buscan soluciones a problemas concretos de interés social, lo que, a su vez, requiere negociaciones continuas con actores no académicos.

- Transdisciplinariedad. La construcción de consensos concretos trasciende el ámbito disciplinar, exigiendo de los científicos mayores dosis de flexibilidad en la adaptación de sus planteamientos y prácticas metodológicas a las particularidades que presente cada objeto de estudio. Se requiere articular teorías, técnicas y procedimientos provenientes de diferentes ámbitos mediante el trabajo en equipo. Prevalecen las zonas hibridas, donde se produce una intersección entre disciplinas e integración de ciencia básica y ciencia aplicada.

- Heterogeneidad organizativa. Esta nueva forma de producir conocimiento ya no se concentra en las universidades y centros de investigación, sino que se multiplican los entornos potencialmente generadores de conocimiento. Pasa a ser predominante la heterogeneidad y la diversidad en lo que respecta a los modos de organización y las formas de trabajo, cuya interacción se canaliza a través de diferentes redes de comunicación.

- Responsabilidad social. Para atender adecuadamente la naturaleza de nuevos problemas con raíz social, los científicos han de intensificar su sensibilidad y compromiso, en especial hacia las implicaciones éticas y políticas de su trabajo. El Modo 2 es, en ese sentido, más reflexivo.

- Control social de la calidad. En el proceso de rendición de cuentas de los resultados se incorporan criterios de índole pragmática y también intervienen otros actores sociales, hasta ahora ajenos al quehacer científico.

En la reformulación posterior de los autores en Re-thinking Science, se introducen nuevos términos e ideas, tales como:

- Contextualización. Diferentes formas de contextualización (débil, media y fuerte) reflejan la diversidad en las relaciones entre ciencia y sociedad. El orden posmoderno es heterogéneo.

- Ágora. Las audiencias heterogéneas se dirigen a los productores de conocimiento en el ágora, que abarca el ámbito político y el mercado. Es el lugar de donde parte la generación y la solución de los problemas y donde se produce la contextualización del conocimiento.

- Comunicación inversa. Se produce desde la sociedad a los productores de conocimiento. La sociedad decide qué es conocimiento. El conocimiento se genera a partir de relaciones fluidas entre el estado, los mercados y la industria.

- Expertos. Actúan en un marco de conocimiento distribuido socialmente atendiendo las necesidades sociales.

- Conocimiento robusto socialmente. Su validez descansa en una amplia comunidad formada por productores, diseminadores, comerciantes y usuarios del conocimiento. 
CUADRO 1: RESUMEN DE LOS RASGOS DE LOS MODO 1 Y MODO 2 DE PRODUCCIÓN DE CONOCIMIENTO

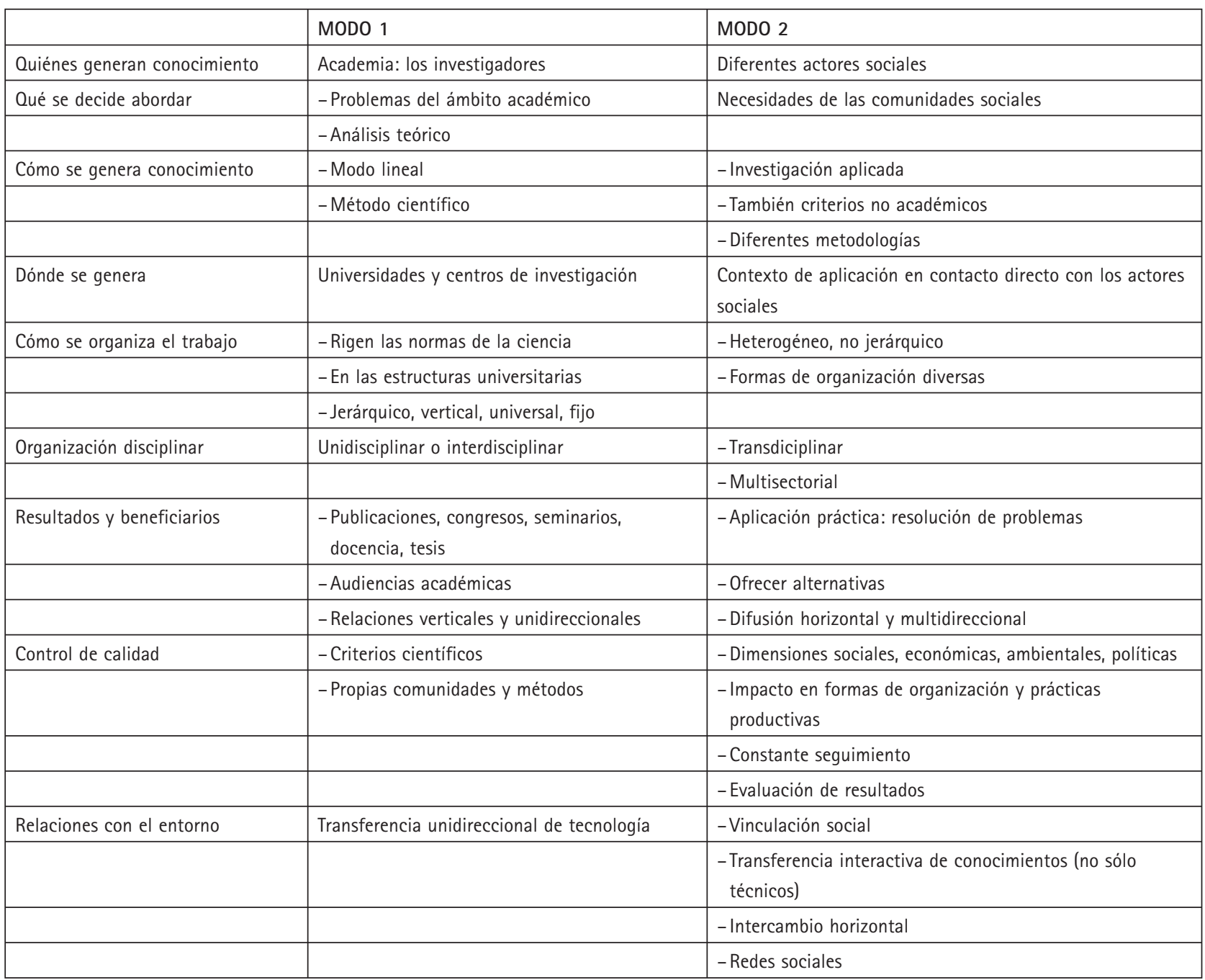

Fuente: elaboración propia a partir de Gibbons et al. (1994) y diversas fuentes.

Esta nueva epistemología alude a la sistematización de la orientación de la investigación hacia la aplicación (Shin, 2002). Pero, básicamente, Rethinking Science transmite en lo fundamental el mismo mensaje que The New Production of Knowledge. Esto es, Nowotny, Scott y Gibbons subrayan el final de la ciencia disciplinaria, del predominio de las universidades y de la investigación de laboratorio. La diferenciación entre conocimiento científico y sociedad da paso a la relevancia de lo social y de las demandas de las empresas. El criterio rector, por tanto, es utilitario
(Albornoz, 2003). La alta valoración social que adquiere la ciencia se debe a que proporciona conocimientos útiles para la vida de los ciudadanos.

\subsection{Críticas y contrapropuestas}

Ante la pregunta de si el Modo 2 representa un nuevo paradigma epistemológico y metodológico, surgen muchas dudas, pues su incidencia en la formulación de políticas públicas contrasta con extendidas y contundentes críticas 
a sus postulados. Críticas que se concentran en torno a los siguiente aspectos:

- Errónea perspectiva histórica. Se cuestiona la identificación temporal del surgimiento del Modo 2 y, por tanto, su supuesta novedad. Para Etzkowitz y Leydesdorff (2000) o Godin (1998), no surgió tras la Segunda Guerra Mundial, ni tampoco es posterior al Modo 1, sino que muchos de los rasgos que Gibbons et al. atribuyen al Modo 2 -tales como la heterogeneidad, el contexto de aplicación y la transdisciplinariedad- ya estaban presentes previamente. De hecho, esos elementos han existido en formas diferentes en distintas disciplinas y sectores. Los intercambios entre el ámbito académico y otros actores sociales, aunque no tan visibles como en la actualidad, no son exclusivos del Modo 2 y, decididamente, ya existían con anterioridad a 1945, como lo atestiguan algunos análisis empíricos. Fuller (2000) sostiene que los dos modos fueron, en realidad, institucionalizados en el siglo XIX. Los autores del Modo 2, además, hacen gala de un sentido de culminación de procesos históricos (Albornoz, 2003), que poco tiene que ver con la realidad, donde los cambios se producen de forma continuada y con extensas ramificaciones. Asimismo, los autores son ambiguos en cuanto al grado de persistencia que cabe esperar del Modo 1 de conocimiento científico sin definir claramente si será sustituido por el Modo 2 o hasta qué punto ambos coexistirán (Hessels y Van Lente, 2008).

- Ausencia de referente teórico y de marco metodológico. Se ha reprochado a los responsables del Modo 2 la falta de un referente teórico claro. Shin (2002) opina que tal contribución no está conectada a ningún marco conceptual, ni en ella se definen los conceptos sociológicos clave. Para Albornoz "la sociedad que asoma de las páginas de Nowotny, Scott y Gibbons es descrita en términos fenoménicos, sin una teoría explícita del cambio en la estructura social" (ibid., 226). Weingart (1997) cree que la falta de profundización teórica les lleva a observar los fenómenos superficialmente y a dramatizarlos. Por otro lado, tampoco aportan un esquema metodológico necesario para desarrollar cualquier programa de investigación (Shin, 2002). No realizan una formulación operativa que se pueda traducir en investigación empírica futura con el correspondiente contraste de hipótesis a través de datos cuantitativos.

- Inexactitudes de contenido. En la literatura se han señalado algunos aspectos que generan dudas sobre su grado de verosimilitud. Así, Godin (1998) estima que es imposible afirmar que el sistema de investigación ha sido alguna vez autónomo y que sólo hoy está en proceso de cambio. Para él la autonomía científica atribuida al Modo 1 es ficticia. Se trata, por el contrario, de una realidad parcial, puesto que, aunque la investigación universitaria era más básica que aplicada, los investigadores también participaban en ocupaciones extraacadémicas. Existirian, además, en el desarrollo científico raíces de componentes hibridos con ramificaciones e intersecciones entre los diferentes ámbitos disciplinares, lo que también pondría en entredicho la dicotomía entre disciplinariedad y transdisciplinariedad.

- Ausencia de evidencias empíricas. Uno de los comentarios más extendidos sobre el Modo 2, estrechamente unido al anterior, es el de que sus tesis no vienen avaladas por el contraste empírico. Shin (2002) subraya ese subdesarroIlo empírico, pues para él las afirmaciones que se vierten no aparecen claramente validadas con hechos. En ningún momento se aportan evidencias concretas. Abundan las preguntas, pero no las respuestas razonadas. De la misma opinión es Godin (1998), para quien los Modos 1 y 2 representan tipos ideales que han de ser corroborados empíricamente. Las conclusiones a las que Ilegan Gibbons et al. y Nowotny et al. carecen de esa base empírica.

- Carácter normativo de la propuesta. Otra de las críticas con mayor consenso dentro de las revisiones que se han hecho al Modo 2 es la de su pretensión normativa, pues describe un conjunto de prescripciones nada obvias correspondientes a un posible programa de política científica que, como tal, es posible orientar en función de los intereses. Godin (1998) lo percibe nítidamente como un alegato político con mezcla de elementos descriptivos y normativos, donde la caracterización de la investigación propia del nuevo modo de producción del conocimiento está supeditada al desiderátum social y político y el tipo de discurso parece más cercano a una ideología política que a una teoría descriptiva. De hecho, si no han faltado los objetores a estos textos es, precisamente, porque "no es fácil percibir si se limita a una descripción fenoménica de tendencias emergentes, o la prescripción de nuevas conductas y pautas organizativas o al desarrollo de nuevas perspectivas cognitivas" (Albornoz, 2003:225). Adicionalmente, las tesis del Modo 2 se han calificado abiertamente de manifiesto político (Shin, 2002). Desde este punto de vista, se interpreta que sus autores creen en un nuevo orden cognitivo y social y tratan de per-

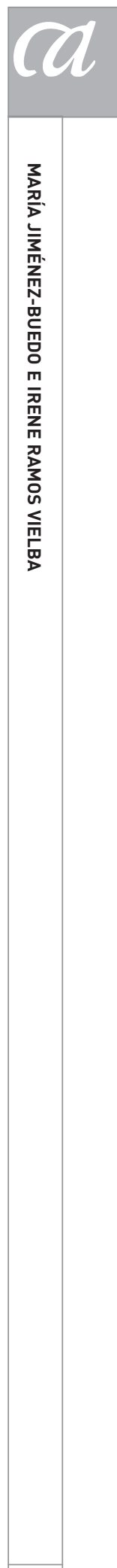


suadir a otros de ello, asimilándose su aportación a una plataforma social y no a un marco de análisis sistemático. En vez de una teoría o un conjunto sólido de información, la nueva producción del conocimiento, tanto el libro como la idea que contiene, se presenta con claros objetivos políticos. Sin embargo, los comentarios sobre la carga normativa de The New Production of Knowledge, para Hessels y van Lente (2008), no son del todo acordes con el contenido del libro, sino que una lectura detenida del mismo pone de relieve que los autores no tenían intenciones normativas explícitas (en la primera página del mismo Gibbons et al. especifican que sus pretensiones son sobre todo descriptivas), aunque sí reconocen que deberían haber sido más cuidadosos al demostrarlo. Defienden que la forma en la que se presenta el mensaje, no resta validez al contenido descriptivo y conceptual del Modo 2, que mantiene intacta su utilidad.

- Dificultades para extrapolar la tesis. Cuando Nowotny, Scott y Gibbons destacan la coevolución de la ciencia y la sociedad, no se puede interpretar un sentido universal (Pestre, 2003), sino que se refieren a una sociedad concreta, donde los contornos de los estados-nación se han difuminado y tampoco existe una nítida demarcación entre la esfera pública y la privada. En ese nuevo contexto, el estado ha disminuido su responsabilidad en la política social, predomina la imagen política y los avances en los marcos regulatorios de la ciencia y la tecnología se fundamentan en amplias negociaciones entre diferentes actores sociales (Albornoz, 2003). En consonancia con esto, Vara y Hurtado de Mendoza (2007) advierten que los autores firmantes del Modo 2 están implicados activamente en la elaboración de políticas públicas, por lo que representan el establishment en sus países de origen y en la Unión Europea. Critican la ausencia de los países periféricos, a los que no se les presta atención alguna, por lo que son contrarios a que se traslade a otros escenarios donde las necesidades y las reglas de juego son diferentes.

En 2003 Nowotny, Scott y Gibbons ${ }^{2}$ responden a las críticas que se habian vertido hasta entonces sobre los dos textos, insistiendo en la vigencia de su tesis sobre la enorme transformación que ha sufrido la producción de conocimiento y el proceso de investigación. El Modo 2 -reiteran- no sólo es un concepto (que puede ser objeto de manipulación), sino que también lo defienden como proyecto y ejemplo de distribución social del conocimiento. Consideran que el escepticismo frente al mismo procede de aquellos que más tienen que perder en la nueva situación: los investigadores establecidos en disciplinas e instituciones, y advierten que las ideas contenidas en el Modo 2 no pueden ser codificadas en las formas tradicionales de las publicaciones científicas. Argumentan que sus dos libros fueron escritos como ensayos reflexivos, y no como estudios empíricos, con la intención de crear un nuevo lenguaje de investigación que permitiera el análisis detallado de las tendencias que ellos describen. Nunca pretendieron que la idea de Modo 2 se convirtiera en una etiqueta para la ciencia aplicada. Aceptan que se eche en falta una discusión más profunda de las dinámicas sociales y que se opine que The New Production of Knowledge ofrece una visión posmoderna de la investigación. Sin embargo, mantienen que el segundo libro -Re-thinking Science- desarrolla suficientemente las nociones iniciales, rellena los huecos argumentativos y ofrece más explicaciones teóricas, para terminar concluyendo que el debate sobre el Modo 2 continúa abierto, pues ésa es su naturaleza.

Precisamente porque el segundo libro no supone poner en entredicho ninguno de los planteamientos originales, sino un intento por dotarlo de mayor anclaje explicativo, las críticas volcadas sobre el Modo 2 mantienen su vigencia. En todo caso, es indudable la contribución de este enfoque al debate respecto a los cambios acaecidos en las últimas décadas en las interacciones ciencia-sociedad. Nadie niega la coevolución de la ciencia y la sociedad que proclaman en Re-thinking Science; cuestión diferente son las diferencias en las valoraciones acerca de la complejidad y las contradicciones asociadas a dicha transformación. El contraste entre Modo 2 y Modo 1, como representación de modelos ideales, no genera interés en lo que respecta a su contenido histórico. Frente a esa polarización, despierta mayores adhesiones -especialmente, dentro de la Filosofía y Sociología de la Ciencia- las tesis que defienden la presencia a lo largo de la historia de formas heterogéneas de producción de conocimiento vinculadas a diferentes instituciones y valores sociales. Para Hessels y van Lente (2008) resulta más válido concebir el Modo 1 y el Modo 2 como los extremos de un continuo en el que situar diferentes prácticas de producción de conocimiento, en vez de dos categorías mutuamente excluyentes. Por otro lado, la explicación de cómo se produce el cambio social continúa siendo un objetivo de enorme interés para historiadores y sociólogos. Pero para poder analizar en profundidad la in- 
cidencia de los factores sociales en la actual configuración de la producción de conocimiento es preciso desprenderse de una visión demasiado idealizada y optimista sobre los cambios que afectan a la ciencia. Sólo así podrá aflorar el amplio abanico de elementos que entran en contraposición en ese proceso, no exento de efectos difíciles de predecir.

A pesar de la diversidad de planteamientos que pudieron incidir en el desarrollo de los contenidos de The New Production of Knowledge, el contexto en el que se realizó el informe que dio lugar a las tesis del Modo 2 recibe influencias de las corrientes postmodernas en los estudios de la ciencia. Quizá debido a ese influjo la pretensión de estos autores -tal y como ellos mismos reconocen- era de carácter ensayístico, sin el propósito de que sus tesis fueran comprobadas mediante el contraste empírico. Respondería, de esa manera, su ejercicio a lo que Boudon (2004) denomina "sociología expresiva", identificando bajo esa etiqueta a aquellas obras que han destacado durante un tiempo por la forma en que expresaban situaciones de la vida social.

\section{Ciencia posacadémica}

La conocida noción de "ciencia posacadémica" se debe a John Ziman. Las elaboraciones más extensas aparecen en los conocidos libros Prometeus Bound: Science in a Dynamic Steady State (1994) y, sobre todo Real Science: What it is, and What it Means (2000), aunque las formulaciones iniciales se encuentran ya en algunos informes y artículos realizados por el autor a finales de los años 1980. Con este término Ziman caracteriza a la empresa científica contemporánea como un sistema en transformación que comparte muchos de sus rasgos con lo que Gibbons y sus coautores han denominado Modo 2 de producción del conocimiento.

John Ziman, tras una brillante carrera en física y algunas experiencias de colaboración en gestión universitaria, transitó gradualmente hacia el estudio de los determinantes sociales de la ciencia que combinó con posiciones militantes con respecto a la reflexión pública del papel cambiante de las ciencias y los científicos en las sociedades posindustriales. Si bien sus primeros trabajos de Filosofía de la Ciencia datan de finales de los años sesenta (Ziman, 1968), y aunque ya en esa década había colaborado en algunos informes para varias oficinas oficiales, no fue hasta los años setenta, cuando abandonó definitivamente la investigación en física, que las reflexiones de Ziman sobre los determinantes sociales de la ciencia maduraron y constituyeron el núcleo de su actividad. Cuando escribe sus trabajos sobre ciencia posacadémica Ziman era ya bastante conocido por sus ensayos sobre las características de la "gran ciencia", ensayos éstos con un importante componente de divulgación.

Sus elaboraciones más relevantes en la línea de pensamiento que aquí se trata se inician en los años ochenta, cuando Ziman es nombrado primer director del Science Policy Support Group. Se trata de un grupo de asesoramiento independiente creado bajo los auspicios de varios Research Councils británicos (especialmente el de ciencias sociales y económicas), con el objetivo de reunir información e impulsar programas de estudio estratégicos en asuntos de política científica. En este foro confluyen entonces numerosos especialistas de varias instituciones y especialidades que dan lugar a un importante grupo de informes de diagnóstico sobre el estado de la I+D, algunos de ellos realizados en estrecha colaboración con SPRU ${ }^{3}$. Es en este contexto en el que surgen varios escritos que ya contienen los conceptos seminales de Ziman sobre el estado de la I+D que posteriormente desarrolla en sus libros, sobre todo los informes Science in a Stedy State (Ziman, 1997) y Restructuring Academic Science (1989), y que tienen una especial trascendencia en el ámbito político.

De esta forma, Ziman constituye en esos años un importante referente público en los debates acerca de las reformas en los sistemas de $I+D$, especialmente intensas en los países anglosajones en esa época. De hecho, algunas de las elaboraciones de Ziman en el SPSG son al mismo tiempo un diagnóstico y una llamada de atención a los límites de las reformas sobre el sistema científico y universitario que lleva a cabo el gobierno británico en la década de los ochenta ${ }^{4}$. Sin embargo, el calado de su obra en la teoría social referida a I+D es algo más limitado, posiblemente porque ni la sensibilidad ni el método de trabajo de Ziman se corresponden con el de los corrientes científico-sociales dominantes en este campo (Ravetz, 2005) (Lipton, 2003).

En Prometheus Bound: Science in a Dynamic Steady State (1994), John Ziman examina la ciencia que comienza a 
enfrentarse a un futuro en el que los recursos ya no crecen o lo hacen a un ritmo mucho más lento que en décadas anteriores. El crecimiento exponencial de los recursos dedicados a la ciencia que caracterizó a las décadas posteriores a la Segunda Guerra Mundial y que había acabado siendo tomado como norma por unos científicos acostumbrados a sus privilegios termina, y la ciencia empieza a competir por los medios económicos con otras prioridades de unos Estados occidentales que muchas veces se enfrentan a déficit presupuestarios que hacen necesaria la revisión de la gestión pública de la actividad científica. Compartiendo gran parte de las ideas de Nowotny et al. acerca de las transformaciones de los sistemas de I+D y del modo de producción científica, Ziman dedica una atención mayor -quizá motivado por cuestiones biográficas- a las consecuencias que estos cambios tienen para los investigadores. En este sentido, y como veremos más adelante, una de las ideas de Ziman más reconocidas y citadas en la literatura la constituye su enmienda a las tesis mertonianas acerca de las fuentes de legitimación de la actividad de los científicos en las sociedades post-industriales.

La motivación central de la obra de Ziman dedicada a la noción de ciencia posacadémica es la de aportar una caracterización de lo que para el autor constituye una "transformación radical, irreversible y mundial de la manera en que la ciencia se organiza y ejecuta" (Ziman, 2000, 7), aunque Ziman no es particularmente exhaustivo al explicar las causas de esta transformación. El principal mecanismo detrás del cambio es, básicamente, el aumento de la competición por los fondos destinados a investigación en una economía caracterizada por una creciente competencia de mercado. Ante estas presiones, la ciencia académica se ha contaminado de las prácticas de la ciencia industrial para dar lugar a un sistema de innovación que él Ilama postacadémico ( $y$ otras veces posindustrial o de "estado estable") y que se caracteriza por un mayor grado de burocratización, un mayor énfasis en la utilidad directa de la producción científica, la necesidad de rendir cuentas ante la sociedad y por transformar la ciencia en una proyecto colectivo, en el que el individualismo de los científicos académicos tradicionales ha sido definitivamente socavado. La colectivización de la ciencia, concepto fundamental en el retrato que Ziman traza de la ciencia posacadémica, se refleja en el hecho de que los científicos de hoy en día tienen menos autonomía en la elección de sus problemas de investigación, ya que los objetivos de la investigación son establecidos por diversas organizaciones no científicas $y$, además, se ven ahora obligados a trabajar en un marco social más organizado en el que tienen que demostrar su experiencia como miembros de equipos de investigación.

De esta forma, según Ziman, la colectivización de la ciencia no sólo ha cambiado la función social externa de la ciencia, sino que ha alterado también la sociología interna del colectivo de investigadores. En este punto, el autor parte, transformándola, de la caracterización clásica que en 1942 Robert Merton hiciera de las normas que gobernaban la actividad de la comunidad científica, conocida por su acrónimo en inglés CUDOS, y que coincide fonéticamente con el término griego kudos, traducible por renombre o fama derivada de un logro o descubrimiento. Las conocidísimas normas mertonianas que resumen los principios de la buena ciencia son el Comunalismo, el Universalismo, el Desinterés, la Originalidad y el E(S)cepticismo. Merton caracterizó así el ethos de la ciencia por el hecho de que los resultados de la investigación pertenecian a la comunidad científica en su totalidad y debian ser compartidos de forma desinteresada por aquellos que buscaran ampliar las fronteras del conocimiento mediante el trabajo original y heterodoxo. Para Ziman, estas normas son las que caracterizan la tradición de la ciencia académica, y sobre todo, la ciencia básica, pero no sirven para entender las dinámicas sociales que rigen la ciencia postacadémica. La ciencia postacadémica, en la medida en que está fuertemente condicionada por los intereses industriales y comerciales, queda caracterizada por su carácter apropiable o interesado, por estar sujeta a la autoridad de los gestores, por proyectos realizados por encargo, y llevada a cabo por expertos locales (en inglés: Propietary, Local, Authoritarian, Commisioned and Expert). El acrónimo inglés PLACE, traducible por posición o puesto, daría así cuenta de lo que el investigador que se dedica a la ciencia industrial consigue al trabajar en esta rama de la ciencia (Ziman, 1995). Se ha interpretado, y de ello en parte es responsable el propio Ziman, que PLACE constituye asi un ethos prácticamente antitético al mertoniano, aunque se hace evidente que contraponer CUDOS a PLACE de una forma directa es un ejercicio algo forzado pues las nociones que constituyen el segundo de estos acrónimos no tienen el carácter normativo de los principios de Merton, sino que son, más bien, características que, en todo caso, describirían, de forma no particularmente exhaustiva ni analítica, algunas de las características de algunas de las ramas investigadoras de la ciencia posacadémica. 
Al igual que en el caso de las tesis acerca del Modo 2, la noción de ciencia posacadémica de Ziman no se apoya sistemáticamente en el análisis empírico de los sistemas de I+D contemporáneos (Hicks et al, 1996, Krige 1996) ni en la comparación de indicadores a lo largo del tiempo. El estilo del trabajo de Ziman es fundamentalmente ensayístico $y_{1}$ en ocasiones, incurre en contradicciones entre algunas de sus propuestas. Por ejemplo, y al igual que sucede en el caso del Modo 2 de producción científica, Ziman es ambiguo con respecto a si la ciencia posacadémica tiene que ser vista como un tipo de actividad que convive con las formas tradicionales o si, por el contrario, las sustituye (Hessels y Van Lente, 2008). Pero Ziman no es sólo ambiguo con respecto a si la ciencia postacadémica sustituye o no a la ciencia académica, sino con respecto, y esto quizá sea más espinoso, a la posibilidad de convivencia entre ambas. Ziman sostiene, a un tiempo, que la ciencia postacadémica supone una revolución sin precedentes, implacable e irreversible $y$, por otro lado, que el término no implica un rechazo o una revocación de los objetivos tradicionales y que indica más la continuidad que la diferencia, porque la ciencia académica y posacadémica se funden entre sí (Ziman, 2003). Se trata por tanto de una contribución sugerente, inserta en una tesis más general acerca de los determinantes sociales de las normas epistémicas de los científicos, pero que adolece de una formulación empírica, o siquiera analíticamente fuerte, que cabría esperar de una caracterización sociológica de las transformaciones contemporáneas en la ciencia.

En cuanto a las implicaciones normativas de la transformación de la ciencia contemporánea, Ziman muestra una particular preocupación acerca de la posibilidad de que la ciencia posacadémica, al impedir que los científicos pasen de elegir problemas que les resulten intrínsecamente interesantes en favor de otros dictados por los intereses del gobierno o la industria, genere un tipo de conocimiento más fragmentado y menos integrado en el sistema general. Pero, sobre todo, le preocupa que en un régimen donde el patrocinio es más directo la posibilidad de sesgos se amplíe (Segerstrale, 2001). Sin embargo, y dada la posición mediadora de Ziman en las guerras de la ciencia entre aquellas caracterizaciones idealizadas y prescriptivas, por un lado (que él resume bajo la rúbrica de "la leyenda"), y otras nociones más desencantadas próximas a enfoques constructivistas (Schimank, 2001), el autor entiende que la ciencia se caracteriza, sobre todo, por su habilidad de adaptación constante a un entorno cambiante. Aboga, por tanto, por una epistemología evolucionista (en la línea de algunos trabajos de Karl Popper, Donald Campbell y otros) en la que la ciencia avanza gracias a los principios evolutivos de "variación ciega" y "retención selectiva" en un proceso abierto.

\section{Ciencia posnormal}

Paralelamente en el tiempo -inicios de los años noventa- surge el enfoque de la "ciencia posnormal". La autoría de este enfoque se debe a la contribución conjunta de Silvio Funtowicz ${ }^{5}$ y Jerome Ravetz ${ }^{6}$, quienes desde 1992 han venido desarrollando sus postulados en diversos artículos científicos -especialmente el publicado en Futures en 1993- relacionados con la resolución de problemas medioambientales ${ }^{7}$. Los planteamientos de fondo de estos autores aceptan la coexistencia de distintos modos de producción científica, adoptando un punto de vista que entiende la forma de funcionamiento de la I+D como un sistema complejo, y que está alejada de supuestos reduccionistas y mecanicistas. En esos contextos contemporáneos de gran complejidad la ciencia se ve limitada para proporcionar respuestas definitivas, lo cuál constituye la situación que da lugar a la diversificación de formas de producir conocimiento.

El nombre de ciencia posnormal deriva de la aportación de Thomas Kuhn, quien en 1962 en The Structure of Scientific Revolutions, introduce el concepto de "ciencia normal" como parte de su teoría sobre el progreso del conocimiento científico mediante consecutivos cambios de paradigma. Dichos cambios se producen por la alternancia de períodos de estabilidad -ciencia normal-, donde se han acordado una serie de reglas bajo las cuales la ciencia intenta dar soluciones a los enigmas que se plantean, con otros períodos de crisis -ciencia revolucionaria- en los que las reglas hasta entonces comúnmente aceptadas se ponen en cuestión y se resuelven las contradicciones generadas. La ciencia normal de Khun se centraría, por tanto, en la resolución de problemas sin poner en entredicho el marco analítico general o los contornos del paradigma vigente en un momento determinado. Tal es la tarea a la que el colectivo de los científicos consagra la mayor parte de su tiempo y dedicación, sosteniendo y alimentando el dogmatismo científico. Sin embargo, la investigación disciplinaria 
tradicional encuentra importantes limitaciones cuando la sociedad ha de resolver asuntos de carácter transdisciplinar, por ejemplo, en el caso de las políticas públicas sobre riesgos medioambientales, que son transnacionales y afectan a varias generaciones. En esos casos no existe un marco incuestionable y ahí es donde surge la ciencia posnormal (van der Sluijs, 2007). No cabría, sin embargo, identificar la ciencia posnormal con la ciencia extraordinaria kuhniana, es decir, con los periodos de crisis de un paradigma, porque a diferencia de los períodos revolucionarios de Kuhn, no se refiere a situaciones transitorias que con el tiempo darán paso a un nuevo período de ciencia normal. Se trata de estados permanentes, en los que la incertidumbre proviene de las características mismas de los sistemas implicados, y se trataría además, de situaciones cada vez más comunes (Diéguez, 2006).

El punto de partida de la ciencia posnormal de Funtowitz y Ravetz es el reconocimiento de que la incertidumbre es inherente a los sistemas complejos. Encontrándonos inmersos en ellos es necesario tomar decisiones, incluso antes de contar con evidencias científicas $y$, sin embargo, el impacto potencial de tales decisiones es muy grande, a lo que se une la existencia de valores en disputa. Por otro lado, la base del conocimiento se caracteriza por las incertidumbres, la multicausalidad y el entendimiento imperfecto. De ese modo, el incremento de la investigación no implica, necesariamente, una reducción de la incertidumbre y es siempre preciso considerar la existencia de consecuencias imprevistas. En los análisis científicos predomina el uso de modelos, escenarios, asunciones y extrapolaciones, pero muchos valores se esconden, precisamente, tras el propio planteamiento de los problemas, los indicadores que se eligen o las asunciones que se realizan (van der Sluijs, 2007). La ciencia posnormal -según Funtowicz y Ravetz- sería la situación en la que se encuentra la investigación científica cuando ha de enfrentarse a situaciones que implican una incertidumbre profunda, una realidad ambigua y unos criterios confusos de calidad. Así, en un problema que conlleva riesgos ambientales globales los hechos son inciertos, existen valores en disputa, el riesgo es alto y las decisiones urgentes. En tales circunstancias una metodología simple no sirve, sino que hay que elegir la estrategia científica de resolución de problemas más adecuada. Las opciones entre las estrategias que estos autores proponen las representan en un diagrama en cuyos ejes ubican el riesgo en las decisiones -lo que se pone en juego- y la incertidumbre de los sistemas, atributos opuestos a lo que tradicionalmente se pensaba que caracterizaban a la ciencia, a saber, su certeza y neutralidad valorativa. Este diagrama muestra la interacción de los aspectos epistémicos (conocimiento) y axiológicos (valores) de los problemas científicos y ahi reside la novedad de su planteamiento.

En este diagrama se representan la ciencia aplicada, la consultoría profesional y la ciencia posnormal, que surgen - por ese orden- según aumenta el grado de intensidad de la incertidumbre y de los riesgos de un problema determinado. Cuando ambos son bajos, nos encontramos con la ciencia aplicada, donde la habilidad profesional y el conocimiento especializado siguen siendo eficaces para la resolución. En ese primer estadio, las incertidumbres de los sistemas se sitúan en los aspectos técnicos a los que se puede hacer frente siguiendo los procedimientos estándar. Si ambos -riesgos e incertidumbres- son de nivel medio, entonces la aplicación de las técnicas rutinarias no es suficiente, se necesita combinarlo con otras habilidades y destrezas porque surgen aspectos más complejos -como la fiabilidad de las teorías que se utilizan-. La incertidumbre aparece en el nivel metodológico. Por eso, aunque la consultoría profesional incluye la ciencia aplicada, los problemas a los que ha de hacer frente requieren una estrategia diferente para la resolución completa. "La consultoría profesional exige creatividad, así como dispo-

Figura 1. Diagrama de estrategias para la resolución de problemas.

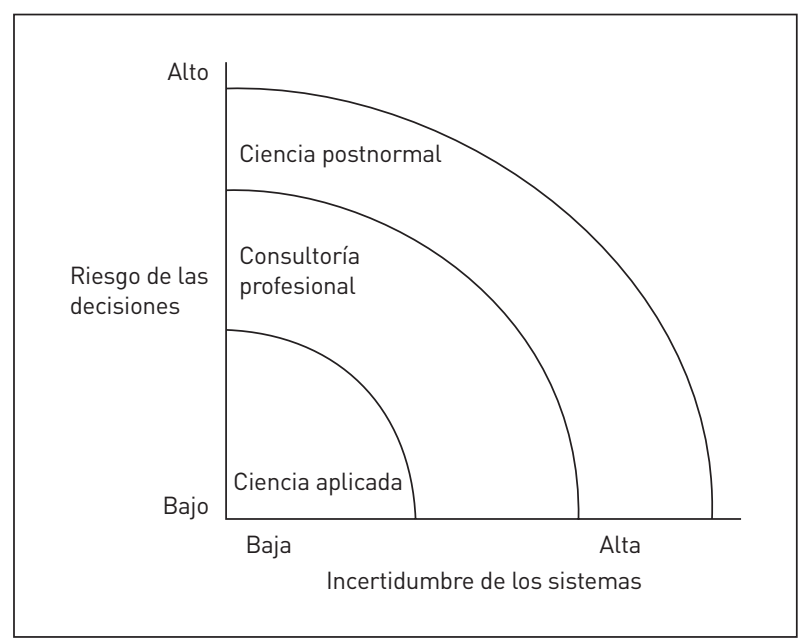

Fuente: Funtowicz y Ravetz, 2000. 
nibilidad para captar las situaciones nuevas e inesperadas y asumir la responsabilidad por sus resultados" (Funtowicz y Ravetz, 2000, 44). Por último, el tercer tipo de estrategia de resolución de problemas se produce cuando las incertidumbres son elevadas y es mucho lo que se pone en juego. En esa situación los afectados persiguen objetivos contradictorios entre sí. Como ocurría en el caso anterior, la ciencia posnormal abarca los estadios inferiores -consultoria profesional y ciencia aplicada- pudiendo formar parte de la resolución, pues no todos los aspectos implicados conllevan una incertidumbre alta o valores en conflicto. Sin embargo, las tareas profesionales o los ejercicios de investigación aplicada no pueden dominar el proceso de toma de decisiones. La ciencia posnormal aparece cuando las incertidumbres son de tipo epistemológico o ético, o cuando lo que se pone en juego en las decisiones refleja intereses en conflicto entre aquéllos que arriesgan algo en el juego. Por lo tanto, en la ciencia posnormal coexisten la gestión de la incertidumbre con la observación de los fenómenos bajo el prisma de los fundamentos teóricos y todo ello, a su vez, con la pluralidad de perspectivas y compromisos (van der Sluijs, 2007).

La ciencia posnormal implica investigación en la intersección entre ciencia y política donde las incertidumbres y la carga valorativa son cruciales. Puede ser interpretado como el proceso de elaboración de una política pública en el que se incorporan prioridades, personas, procedimientos, productos y análisis postnormal, extendiéndose también a las fases de implementación y seguimiento. Consecuentemente, dependiendo del contexto en particular, la tarea tendrá más componentes de investigación o de creatividad innovadora de carácter técnico-social. Todo el proceso es en sí un sistema complejo donde se interrelacionan elementos naturales con otros técnicos y sociales. Efectivamente, en nuestra sociedad moderna los científicos y otros profesionales asumen determinados liderazgos en aspectos técnicos que sirven de apoyo a la toma de decisiones políticas. Sin embargo, se viene comprobando en las últimas décadas que únicamente su pericia no resulta suficiente para resolver grandes dilemas que afectan a la ciencia en el mundo actual. Cuando los riesgos no son cuantificables o el daño potencial puede ser irreversible, es imposible hacer recaer en las metodologías hasta ahora utilizadas y las formas tradicionales de conocimiento la orientación de las políticas públicas. Ése es el espacio de la ciencia posnormal, donde la práctica científica puede afrontar los desafíos implícitos en los contextos complejos en los que colisionan pluralidad de actores y valores. Para ello se articula -y ésta es la caracteristica más destacable de la ciencia posnormal- la participación pública.

Ciertamente, la propuesta más novedosa y reseñable que aporta el enfoque de la ciencia posnormal consiste en la denominada "extensión de la comunidad de pares" como reflejo de la incorporación de la participación pública en la resolución de los problemas. Existen actualmente numerosas iniciativas dirigidas a incorporar a amplios círculos de colectivos en los procesos de toma de decisiones y también en su posterior implementación -por ejemplo, en cuestiones relativas al medio ambiente o la salud-. Sin embargo, la implicación en la ciencia postnormal de las partes interesadas no sólo se asocia con la participación democrática, pues se considera que el planteamiento de Funtowicz y Ravetz tiene otras connotaciones adicionales. Así, frente a la entidad de los nuevos problemas, el mantenimiento de la calidad depende de un vasto diálogo entre los afectados, por lo que la extensión de la comunidad de pares -0 comunidad de pares ampliada- no la componen únicamente aquellas personas con acreditación institucional, sino todos aquellos meros interesados en tomar parte en la resolución de un asunto concreto. $Y$, de ese modo, se incrementa la exigencia de rendimiento de cuentas a los gobiernos en cuanto a la gobernanza de la ciencia y la tecnología (Constanza, 2008). Es más, de acuerdo con la visión de la ciencia posnormal, la calidad de los inputs científicos para los procesos de elaboración de las políticas públicas deben desempeñarlos dichas comunidades ampliadas. Según la aportación de Funtowicz y Ravetz la dinámica de la resolución de los problemas en la ciencia posnormal conlleva la inclusión de un conjunto cada vez más creciente de "participantes legítimos" en el proceso que sirve para asegurar la calidad de los inputs científicos, tal sería el caso de las personas directamente afectadas por un problema ambiental, pues tendrán una conciencia más profunda de sus sintomas y un interés más apremiante que aquéllos a los que no les concierne directamente.

En los últimos años es creciente la utilización de las comunidades ampliadas de pares, cuando las autoridades no ven una buena salida a los problemas, o cuando se sabe de antemano que sin un consenso amplio las decisiones no podrán salir adelante. Adquieren formas varias como jurados ciudadanos, grupos de discusión, conferencias de 
consenso, talleres de escenarios, talleres de ciencia, etc. (Del Moral y Pedregal, 2002). El elemento común radica en que estas comunidades ampliadas valoran la calidad de las propuestas, incluida la parte científica. Demuestran su competencia combinando determinados conocimientos científicos con su propia situación. Sus veredictos contienen fuerza moral $y$, por ende, también influencia política. No son receptores pasivos de los materiales que les proporcionan los expertos, sino que aportan otra serie de conocimientos y de datos más ligados a sus propias experiencias y trayectorias vitales (Constanza, 2008). El modelo de participación ampliada implica trabajar de forma deliberativa con las imperfecciones $y$, para ello, la ciencia -entendida como la actividad de los expertos técnicos- se convierte sólo en una parte importante de las evidencias utilizadas. Se abre un proceso de diálogo crítico sobre la fortaleza y relevancia de las evidencias, democratizando su interpretación y cuyas conclusiones estarán dotadas de significado político (van der Sluijs, 2007). Funtowicz explica cómo a una mayor diversificación de los modos de producción corresponde también variedad en la formas de evaluación, poniendo como ejemplo el caso de la inadecuación del peer review para las patentes (Sanz, 2007).

En cualquier caso, el alcance de la extensión de la comunidad de pares ha suscitado algunas interpretaciones no coincidentes. Diéguez (2006) contrapone su propia visión a la valoración postmodernista que hace Sardar de la propuesta al someter también a juicio de la comunidad de pares ampliada la evaluación del trabajo científico en sí mismo, contemplando la posibilidad de aplicar diferentes procesos de validación. Esto modificaría sustancialmente el modo de hacer ciencia, sin embargo, no parece deducirse tal pretensión de los planteamientos de la ciencia postnormal que limita la contribución de la comunidad de pares a la participación en la resolución de problemas complejos. Precisamente, lo contrario alimentaría los principales argumentos de los detractores de la ciencia postnormal que creen ver detrás un intento de ignorar los métodos científicos, sustituyéndolos por otras metodologías menos sólidas y, claramente, al servicio de propósitos políticos. Mientras tanto, los defensores de la ciencia postnormal, matizan que no se produce esa sustitución allí donde la ciencia proporciona respuestas satisfactorias, sino que la utilidad de la participación de las comunidades de pares ampliadas se centra en aquellos casos donde las decisiones tienen una gran trascendencia $y_{1}$ sin embargo, la ciencia por sí sola no puede resolverlo, dada la concentración de grandes incertidumbres y las tremendas consecuencias de una resolución errónea. En esas situaciones la ciencia contribuye a enmarcar y analizar el problema, pero la inclusión de la comunidad ampliada redunda en beneficio del proceso y del resultado final.

Diferentes revisiones valorativas de la ciencia posnormal han observado algunas similitudes con el Modo 2 de producción del conocimiento. Aun apreciando mayor carga prescriptiva en la ciencia postnormal, Hesels y van Lente (2008) observan que los dos enfoques prestan atención al incremento de interacciones más allá de fronteras disciplinares y organizativas, son partidarios de una mayor flexibilidad general y consideran criterios adicionales en la medición de la calidad. Sin embargo, existe -en su opinión- una gran diferencia en cuanto al ámbito al que se refieren, puesto que la ciencia postnormal se centra en la investigación que sirve de apoyo a las políticas públicas y deja fuera las interacciones universidad-empresa. Las empresas pueden participar como una parte interesada en un problema específico, pero no por su potencial como coproductores de conocimiento. El interés por la función pública de la investigación sitúa, sin embargo, a la ciencia posnormal cerca de la idea de Nowotny et al. (2001) sobre el conocimiento socialmente robusto. Preguntado por la vinculación con el Modo 2, Funtowicz señala:

Nuestra propuesta es enunciada habitualmente junto al Modo 2 de producción de conocimiento (de Gibbons y Nowotny), pero hay que distinguir ambas ideas. Si como nueva forma de producción científica se tiene en mente el Modo 2, lo que se está haciendo es destacando el hecho de que hay un nuevo tipo de foco para la producción científica que transforma el modo anterior (dado, por ejemplo, en los ámbitos académicos). En cambio, cuando nosotros hablamos de "Ciencia postnormal" lo que hacemos es destacar que la complejidad está dada, precisamente, por la variedad de modos de producción científica y cómo los recursos, el prestigio, el poder... se establecen de distinta forma en uno u otro de estos modos (Sanz, 2007, 342).

Albornoz (2003), por su parte, entiende que la disimilitud más importante entre los dos enfoques se sitúa en el hecho de que Funtowicz y Ravetz, cuando abordan el problema de la incertidumbre y la complejidad como desafíos crecientes para la ciencia, no se refieren a un nuevo tipo de cono- 
cimiento. La ciencia posnormal conlleva -más bien- una nueva forma organizativa, pero sin por ello cuestionar los fundamentos de la ciencia tradicional. Ésta se contextualiza y actualiza en un marco donde ahora participan otros actores. Finalmente, cabe destacar que la ciencia posnormal -a diferencia del Modo 2- sí ha dado lugar a un programa de investigación y, de hecho, se han desarrollado bajo este enfoque diferentes herramientas metodológicas -Tools for Knowledge Quality Assesment: NUSAP, quality checklists (van der Sluijs, 2007)- y se han aplicado sus planteamientos a la resolución de problemas de indole medioambiental (energías, transgénicos, cambio climático, etcétera), para lo cual los científicos han desarrollado nuevas capacidades profesionales y se han habilitado procesos deliberativos políticamente significativos.

Los trabajos de Funtowicz y Ravetz están relacionados con la corriente dentro los Science and Technology Studies (STS) que defiende la superación definitiva de la contraposición "internalismo-externalismo", lo que implica que los filósofos de la ciencia se ocupen cada vez más de aspectos sociales y políticos, y no tanto de cuestiones de método, teorías o epistemología. Desde este punto de vista, el enfoque de los STS consiste en observar cómo los valores sociales, políticos y culturales afectan a la investigación y a la innovación tecnológica $y$, a su vez, cómo esto afecta a la sociedad y a las formas culturales. Tal es el punto de vista que privilegia la tradición de la que provienen estos autores, y por ello quizá sus estudios han derivado mucho más en la relación ciencia-sociedad (movimientos sociales y políticos, espacios culturales) y no tanto en aspectos de organización y cambio de las organizaciones científicas tales como universidades, centros públicos, organismos de financiación o dirección política, empresa, etc. Todo esto parece tener como consecuencia que la ciencia posnormal sea el enfoque que más ha evolucionado como programa de investigación, ya que existen una serie de personas y centros que hacen investigaciones empíricas en ese ámbito y contribuyen a aumentar la densidad y a redefinir y extender los problemas que Funtowicz y Ravetz plantean.

\section{Conclusiones}

En un contexto de crecimiento de la producción científica y de cambio en las fuentes y formas de financiación de la ciencia como el que vivimos sólo cabe esperar que los sistemas de I+D sufran transformaciones, muchas de las cuales son, a todas luces, profundas e irreversibles. Para los enfoques de los que nos hemos ocupado en este artículo estas transformaciones han dado lugar a nuevas formas de producción científica y coinciden en señalar varios rasgos que a juicio de Ziman, Funtowicz y Ravetz, y Gibbons y sus colaboradores, dan cuenta de cambios radicales en la manera de hacer ciencia.

En primer lugar, la producción de conocimiento se hace reflexiva y se somete al principio de rendición de cuentas ante la sociedad. De esta forma, la investigación se orienta cada vez más hacia los valores sociales, económicos y políticos que imperen en cada momento. Así, y como segundo rasgo reseñable, la producción de conocimiento tiene lugar en el contexto de aplicación, con la utilidad concreta para sus clientes o potenciales beneficiarios presente en la mente de los científicos que lo producen. En tercer lugar, los laboratorios industriales, agencias gubernamentales, think tanks y empresas consultoras, han arrebatado el cuasi-monopolio de la producción del conocimiento a las universidades y generan equipos de investigación y redes transitorias centradas o diseñadas para responder a problemas concretos. En cuarto lugar, estos equipos de investigación en los que confluyen científicos de diversos campos han hecho que la división disciplinar de la ciencia da paso a la transdisciplinariedad, más acorde con la ciencia que se genera en los contextos de aplicación. Por último, el control de calidad de la investigación incorpora criterios externos (sociales, políticos y económicos) y no se limita al control interno, a través de los pares, que caracterizaba la autonomía del modelo tradicional de ciencia.

Como hemos visto, los tres enfoques coinciden no solo en señalar estos rasgos como definitorios de las nuevas formas de producción del conocimiento científico, sino en la ambigüedad con la que se refieren al grado de persistencia de las formas tradicionales de ciencia que se están viendo reemplazadas. De esta manera, de la lectura de los trabajos analizados, no podemos deducir hasta qué punto los autores consideran que el Modo 1 de producción de conocimiento, la ciencia académica 0 , la ciencia normal continuarán siendo importantes o serán eliminadas progresivamente por los fenómenos señalados. Esta ambigüedad, que responde al hecho de que ninguno de los tres enfoques especifica el alcance real de los cambios que postulan, se explica por

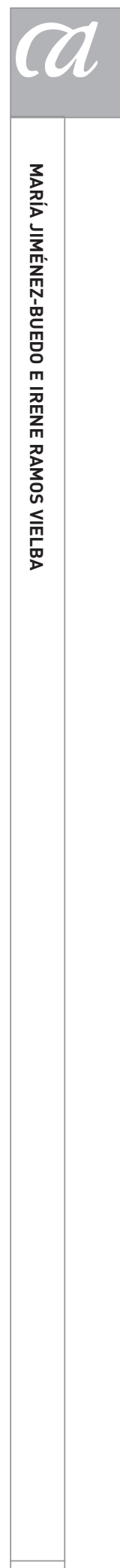

733 
el problema fundamental que comparten las tres nociones de nueva ciencia que analizamos en este artículo: la falta de evidencia empírica sistemática y de especificación de marcos geográficos o temporales concretos con los que comparar la situación actual con un pasado supuestamente definido por las formas tradicionales de organización de la ciencia. Como han señalado los críticos con estos enfoques, el énfasis en la aplicabilidad, la heterogeneidad de las instituciones científicas y la interdisciplinariedad y las transformaciones en las fronteras disciplinarias no son, ni rasgos nuevos en la ciencia, ni rasgos dominantes de los sistemas contemporáneos. Se trata, de hecho, de rasgos esperables de cualquier sistema científico en expansión e inserto en un contexto cambiante.

Un segundo conjunto de rasgos definitorios de las nuevas formas de producción de conocimiento científico se refieren a una mayor participación de los actores sociales en los procesos científicos, lo cual es probablemente un acontecimiento más genuinamente novedoso. No obstante, como ha señalado Weingart (1997), la importancia de estos fenómenos se ciñe a unos pocos campos científicos concretos, como la investigación relativa a cuestiones medioambientales o las evaluaciones tecnológicas. Éstos son, precisamente, los campos científicos que Funtowicz y Ravetz, o Gibbons y sus colaboradores, han estudiado más en profundidad, y esto quizá explica por qué, a falta de un análisis empírico exhaustivo, se hayan aventurado a extrapolar los desarrollos en estos sectores científicos al conjunto del sistema de I+D. Los cambios más llamativos a los que se refieren los enfoques analizados se restringen a un sector relativamente pequeño del sistema científico. Por otro lado, no existe evidencia alguna de que, siquiera en estos campos, se hayan propuesto transformaciones profundas en los criterios epistemológicos de base en la ciencia. Por otra parte, los autores analizados no proporcionan un criterio de demarcación unívoco entre ciencia tradicional y nuevas formas de producción científica. En consecuencia, es difícil evaluar el alcance exacto de la transformación de la ciencia que estos autores postulan y esto hace que una gran variedad de datos empíricos, no necesariamente congruentes entre sí, hayan sido considerados evidencia a favor de estas tesis.

A la vista de todo esto, el balance en cuanto a la influencia e impacto de estos enfoques es ambivalente. De un lado, las ideas subyacentes a las nociones, y las nociones mismas de ciencia posnormal, ciencia posacadémica $y$, especialmente, el Modo 2, han permeado el lenguaje de algunos hacedores de política y de los documentos de trabajo de las instituciones en las que operan, de manera que estas nociones han servido para inspirar y justificar cambios en las políticas públicas. Incluso, también han influido en la forma en que algunos científicos describen su propio trabajo (Cohen et al., 2001). Ahora bien, ¿qué trascendencia han tenido estos enfoques en el campo de los estudios de la ciencia y la tecnología? De los tres enfoques, ha sido el del Modo 2 el que ha encontrado un mayor eco a juzgar por el número de artículos científicos que citan el trabajo de Gibbons et $a l .8$. Si bien es cierto que el enfoque asociado a la ciencia posnormal de Functowicz y Ravetz es menos citado en artículos científicos, si ha dado lugar (al contrario que el trabajo de Gibbons et al.) al desarrollo posterior de una metodología, aunque orientada ésta a la resolución de problemas prácticos relativos a la toma de decisiones colectivas en contextos de incertidumbre y no tanto al estudio de carácter general y exhaustivo de los sistemas de I+D.

En el caso de los trabajos de Ziman, el hecho de que hayan tenido un menor eco es el resultado, en parte, de su estilo heterodoxo y al margen de las corrientes sociológicas contemporáneas, y quizá también del hecho de que comparte más rasgos de los que pareciera a primera vista con la Sociología de la Ciencia de corte funcionalista de Robert Merton. No obstante, no es descartable que aumente el interés por este autor ahora que algunos temas clásicos que habian sido abandonados por la Sociología de la Ciencia (como los sistemas de incentivos, las evaluaciones y el impacto de éstos en las carreras científicas) están siendo recuperados por algunos programas de investigación más centrales en las Ciencias Sociales, como el Institucionalismo.

El mayor mérito de los enfoques aquí analizados, en cuanto que narrativas de alcance medio que describen algunas transformaciones contemporáneas en los sistemas científicos de algunos países desarrollados, puede residir quizá en el hecho de que las nociones de ciencia posacadémica, ciencia posnormal y Modo 2 se han demostrado útiles para generar preguntas de investigación o intentos de dar cobertura empírica a algunas de tesis propuestas. De momento, a medida que estos enfoques generan discusión que da lugar a intentos de evaluar empíricamente las tesis de este grupo de autores, parece cada vez más claro que la ciencia académica, normal o el Modo 1 de producción de 
conocimiento demuestran una enorme capacidad de resistencia, y que siguen siendo fundamentales para entender cómo funcionan los sistemas de I+D.

A la luz de esto, podemos concluir que algunas de las afirmaciones que los enfoques analizados sostienen acerca de cambios radicales en el funcionamiento y organización de la ciencia parecen exagerados. Es posible que el exceso se explique por el hecho de que sirven para corregir (si bien excediéndose en el ejercicio) visiones estereotipadas de la ciencia académica, o lo que Ziman dio en Ilamar "la leyenda" acerca de la ciencia, que circularon profusamente hasta hace algo más de una década. ¿Qué podemos decir, entonces, acerca de si los sistemas de I+D actuales están más allá de la ciencia académica? La respuesta a esta pregunta es, de nuevo, ambivalente. De un lado, es indudable que se han producido importantes transformaciones en la organización interna de universidades y centros de investigación, en la forma en que la universidad y los centros de investigación se relacionan con otros ámbitos sociales o políticos, y en las condiciones de los trabajadores del conocimiento, de forma que las instituciones científicas son sustancialmente distintas a las de hace algunas décadas. Sin embargo, muchas de las características distintivas de la ciencia académica (por ejemplo, la revisión por pares) no sólo se mantienen sino que cobran aún mayor importancia como elemento legitimador de las comunidades científicas ante el público general. Parece pues que asistimos a la coexistencia de una lógica académica y postacadémica dentro de las organizaciones científicas, lo cuál da lugar a situaciones complejas difíciles de gestionar. Muchos de los rasgos de esta complejidad están bien dibujados por aportaciones teóricas como las que aquí se han tratado. Ahora bien, su existencia da lugar a una serie de problemas que una investigación con vocación empírica debe tratar especificando al menos dos cosas: en qué grado se extienden estos rasgos en distintos contextos geográficos y temporales, y cuáles son los mecanismos que explican que se produzca su ocurrencia y, en su caso, la sustitución de una forma dominante por otra.

\section{NOTAS}

Recibido: 15 de octubre de 2008

Aceptado: 20 de diciembre de 2008
1 El proyecto que dio lugar al libro fue encargado por el Swedish Council for Research and Planning, FRN, y tenía como objetivo un ejercicio de prospectiva sobre la situación de las universidades.

2 Se trata de un monográfico de la Revista Minerva dedicado a esta tesis, donde se invita a los autores a realizar una Introducción.

3 Science and Technology Policy Research. Centro de Investigación sobre política científica y tecnológica asociado a la Universidad de Sussex.

4 Sobre el Science Policy Support Group ver http://www.stage-research.net/SPSG/home.html

5 Epistemólogo y matemático, fue Research Fellow en la University of Leeds. Actualmente, investigador y asesor de la European Comission Joint Research Centre, Institute for Systems Informatics and Safety (ISIS) en Varese (Italia).

6 También epistemólogo y matemático, fue profesor de Historia y Filosofía de la Ciencia en University of Leeds e investigador en The Research Methods Consultancy Ltd. de Londres. Es Associate Fellow en el James Martin Institute, Oxford University. Recientemente ha trabajado en la gobernanza de tecnologías emergentes, centrándose en la nanotecnología.

7 En el año 2000 la editorial Icaria tradujo al español algunos de esos artículos. En 2007 Noemi Sanz realizó una entrevista a Silvio Funtowicz, publicada en Argumentos de Razón Técnica, donde defiende la vigencia de los planteamientos posnormales. 8 Hessels y Van Lente (2008). 


\section{REFERENCIAS BIBLIOGRÁFICAS}

Albornoz, Mario (2003): "El problema de re-pensar contextos", Revista CTS, vol. 1, n. ${ }^{\circ}$, pp. 225-230.

Boudon, Raymon (2004): "La sociología que realmente importa", Papers, vol. 72, pp. 215-226.

Cohen, Laurie; McAuley, John y Duberley, Joanne (2001): "Continuity in Discontinuity: Changing Discourses of Science in a Market Economy", Science, Technology, \& Human Values, vol. 26, pp. 145-166.

Costanza, Robert (2008): "Post-Normal Science", en Cutler J. Cleveland (ed.), Encyclopedia of Earth [En linea], Washington, D.C., Environmental Information Coalition, National Council for Science and the Environment. (Última revisión 22 agosto 2008) [ref. de 16 de septiembre de 2008] Disponible en web: <http://www.eoearth.org/article/Post-Normal_Science>

Del Moral, Leandro y Pedregal, Belén (2002): "Nuevos planteamientos científicos y participación ciudadana en la resolución de conflictos ambientales", Documents d'Anàlisi Geogràfica, vol. 41, pp. 121-134.

Diéguez, Antonio (2006): "La ciencia desde una perspectiva postmoderna: Entre la legitimidad política y la validez epistemológica", En Actas de /l Jornadas de Filosofía: Filosofía y politica (Coín, Málaga, 2004), Coín, Málaga, Procure, pp. 177-205.

Etzkowitz, Henry y Leydesdorff, Loet (2000): "The Dynamics of Innovation: from National Systems and 'Mode 2' to a Triple Helix of University-Industry-Government Relations", Research Policy, vol. 29, n. ${ }^{\circ}$ 2, pp. 109-123.

Fuller, Steve (1993): Philosophy, Rhetoric, and the End of Knowledge: The Coming of Science and Technology Studies,
Madison, WI, University of Wisconsin Press. 2nd edition, with James $\mathrm{H}$. Collier, Lawrence Erlbaum Associates, 2004.

Fuller, Steve (2000): The Governance of Science, Buckingham, Open University Press.

Funtowicz, Silvio 0. y Ravetz, Jerome R. (1993): "Science for the Post-Normal Age", Futures, vol. 25, n. ${ }^{\circ}$ 7, pp. 739755.

Funtowicz, Silvio 0. y Ravetz, Jerome R. (2000): La ciencia posnormal: ciencia con la gente, Barcelona, Icaria.

Funtowicz, Silvio 0. y Ravetz, Jerome R.: Post-Normal Science - Environmental Policy under Conditions of Complexity [en línea] [ref. de 19 de agosto de 2008] Disponible en web: <http:// www.nusap.net/sections.php?op=vie warticle\&artid $=13>$

Gibbons, Michael; Limoges, Camille; Nowotny, Helga; Schwartzman, Simon; Scott, Peter y Trow, Martin (1994): The New Production of Knowledge: The Dynamics of Science and Research in Contemporary Societies, London, Sage.

Godin, Benoit (1998): "Writing Performative History: The New New Atlantis?", Social Studies of Science, vol. 28, pp. 465-483.

Hessels, Lauren K. y van Lente, Harro (2008): "Re-thinking New Knowledge Production: A literature Review and a Research Agenda", Research Policy, vol. 37, pp. 740-760.

Hicks, Diana. M. y Katz, J. Sylvain (1996): "Where Is Science Going?", Science, Technology, \& Human Values, vol. 21, 379-406.

Kuhn, Thomas S. (1962): The Structure of Scientific Revolutions, Chicago, The University of Chicago Press, segunda edición ampliada, 1970.

Leydesdorff, L. y Etzkowitz, H. (1996): "Emergence of a Triple Helix of Uni- versity-industry-government Relations", Science \& Public Policy, vol. 23, pp. 279-286.

Lipton, Peter (2003): "The Science of Science. Reviewed work(s): Real Science: What It Is, and What It Means by John Ziman", Notes and Records of the Royal Society of London, vol. 57, pp. 108-111.

Krige, John (1996): "Review: Prometheus bound by J. M. Ziman", Research Policy, vol. 25, pp. 486-488.

Nowotny, Helga; Scott, Peter y Gibbons, Michael (2001): Re-Thinking Science. Knowledge and the Public in an Age of Uncertainty, Cambridge, Reino Unido, Polity Press.

Nowotny, Helga; Scott, Peter y Gibbons, Michael (2003): "Introduction. 'Mode 2' Revisited: The New Production of Knowledge", Minerva, vol. 41, pp. 179-194.

Pestre, Dominque (2003): "Regimes of Knowledge Production in Society: Towards a More Political and Social Reading", Minerva, vol. 41, pp. 245261.

Ravetz, Jerome (2005): "Obituary: John Ziman", en The Guardian, London, Online edition.

Sanz Merino, Noemí (2007): "Gobernanza de la tecnociencia y participación ampliada. Entrevista a Silvio Funtowicz y Javier Echeverria", Argumentos de Razón Técnica, vol. 10, pp. 337-359.

Schimank, Uwe (2001): "Review: Real Science - What It Is, and What It Means - John Ziman", Research Policy, vol. 30, pp. 1343-1344.

Segerstrale, Ullica (2001): "Real Science - What It Is, and What It Means", Endeavour, vol. 25, pp. 79-80.

Shinn, Terry (2002): "The Triple Helix and New Production of Knowledge: Prepackaged Thinking of Science and Technology", Social Studies of Science, vol. 32, n. ${ }^{4}$, pp. 599-614. 
Van der Sluijs, Jeroen (2007): "Post Normal Science, working deliberatively within imperfections" [en línea], lecture at Wageningen University [ref. de de 18 de agosto de 2008] Disponible en web: $<$ http://wurtv.wur.nl/wurtv/viewer. html?path=roadkit3/2007/03/21/1/>

Vara, Ana María y Hurtado de Mendoza, Diego (2007): Negocios... [en línea], Futuro, Argentina [ref. de 18 de junio de 2008]. Disponible en web: <www. pagina 12.com.ar/imprimir/diario/suplementos/futuro/13-1688-2007-0414>

Weingart, Peter (1997): "From "Finalization" to 'Mode 2': old wines in new bottles?", Social Science Information, vol. 36, n. ${ }^{\circ}$, pp. 591-613.

Ziman, John (1968): Public Knowledge: Essay Concerning the Social Dimension of Science, Cambridge University Press.

Ziman, J. (1987): Science in A Steady State: The Research System in Transition, Science Policy Support Group Concept Paper, n. ${ }^{\circ}$, London, SPSG.

Ziman, J. (1989): "Restructuring Academic Science", Science Policy Support Group Concept Paper, n. ${ }^{\circ}$ 8, London, SGPS.

Ziman, John (1994): Prometheus Bound. Science in a Dynamic Steady State,
Cambridge, UK, Cambridge University Press.

Ziman, John (1995): Of One Mind: The Collectivization of Sience, Masters of Modern Physics, Woodbury, NY, R. N. Ubell (ed.), American Institute of Physics.

Ziman, John (1996): "Ciencia y Sociedad Civil", Ciencia, Tecnología y Sociedad, vol. 1, pp. 177-188.

Ziman, John (2000): Real Science: What It is and What It Means, Cambridge, UK, Cambridge University Press.

Ziman, John (2003): ¿Qué es la ciencia?, Madrid, Cambridge University Press. 